\title{
Bifurcation Behavior of a Flexible Rotor Supported on Nonlinear Squeeze Film Dampers without Centralized Springs
}

\author{
ZHU CHANGSHENG ${ }^{\mathrm{a}, *}$ and HEINZ ULBRICH $^{\mathrm{b}}$ \\ a Department of Electrical Engineering, Zhejiang University, 310027, Hangzhou, Zhejiang, P. R. China, \\ ${ }^{\mathrm{b}}$ Institute of Mechanics, Department of Mechanical Engineering, University of Essen, Essen 45127, Germany
}

(Received 17 September 1997; In final form 17 November 1998)

\begin{abstract}
In this paper, the bifurcation behavior of a flexible rotor supported on nonlinear squeeze film dampers without centralized springs is analyzed numerically by means of rotor trajectories, Poincarè maps, bifurcation diagrams and power spectra, based on the short bearing and cavitated film assumptions. It is shown that there also exist two different operations (i.e., socalled bistable operations) in some speed regions in the rotor system supported on the nonlinear squeeze film dampers without centralized springs. In the bistable operation speed regions, the rotor system exhibits synchronous, sub-synchronous, sub-super-synchronous and almost-periodic as well as nonperiodic motions. The periodic bifurcation behaviors of the rotor system supported on nonlinear squeeze film dampers without centralized springs are very complex and require further investigations.
\end{abstract}

Keywords: Rotor, Squeeze film damper, Nonlinear, Bifurcation, Bistable operation, Rotordynamics

\section{INTRODUCTION}

It is well known that there are many nonlinear effects in general rotor systems, such as bearing clearances, seals, fluid-film bearings and squeeze film dampers etc. If the vibration amplitudes of the rotor system are much large, the nonlinear effects are very obvious. There are two nonlinear phenomena due to the nonlinear dynamics: the bistable jumping and the nonsynchronous responses. The former is caused by the multivalueness in the amplitude response curve, the latter is produced by the periodic bifurcation of the rotor motion. Recently, the bifurcation behavior of the nonlinear rotor systems has been paid more attention (Adams and Abu-Mahfouz, 1994; Choi and Noah, 1994; Zhao and Hahn, 1993).

The squeeze film damper is one of the most effective flexible damping elements which have been developed in the past thirty years, but it exhibits

* Corresponding author. Center of Vibration Engineering, Department of Mechanical Engineering, Imperial College, Exhibition Road, London SW7 2BX, England. Tel.: (0044)0171-589-5111, ext. 57078. Fax: (0044)0171-584-1560. E-mail: c.s.zhu@ic.ac.uk. 
high nonlinear behavior in presence of large rotor unbalances and due to improper design conditions. Both the bistable operations and nonsynchronous responses can be observed in experiments, and these problems have been investigated by many investigators both theoretically and experimentally, such as White (1972), Li and Taylor (1987), Rabinowitz and Hahn (1977), Zhao and Hahn (1993), and Zhu and Feng (1987). However, almost all of them are limited to the rotor systems supported on squeeze film dampers with centralized springs in order to simplify the analysis and make an assumption of the journal center describing synchronous circular orbits about the bearing center in analyzing the bistable behavior of the rotor systems. Only a few cover the nonlinear behavior in the rotor systems supported on squeeze film dampers without centralized springs. This paper presents a theoretical investigation of the bifurcation behavior of a flexible rotor supported on nonlinear squeeze film dampers without centralized springs, using the methods of nonlinear dynamics and bifurcation, specifically in terms of rotor trajectories, Poincarè maps, bifurcation diagrams and power spectra, based on the short bearing and cavitated film assumptions.

\section{ROTOR SYSTEM MODEL AND ANALYSIS METHODS}

\section{Rotor System Model}

The rotor system to be investigated in this paper is a Jeffcott rotor symmetrically supported on two identical squeeze film dampers without centralized springs as shown in Fig. 1. In order to analyze this system, the following assumptions are made:

1. the rotating speed is constant and the unbalance of the rotor system is defined at the midplan of the rotor;

2. the rotor mass is lumped at the rotor mid-plan and at the two damper stations;

3. the rotor stiffness is symmetric and the damping at the rotor mid-plan disk due to the air dynamics is viscous;

4. the axial and torsional vibrations of the rotor system and the influence of the rolling bearings are negligible;

5. the oil-film forces of the damper are determined by the Reynolds equation with an incompressible lubricant and the short bearing approximation is applicable;

6. the cavitation results in $\pi$-film.

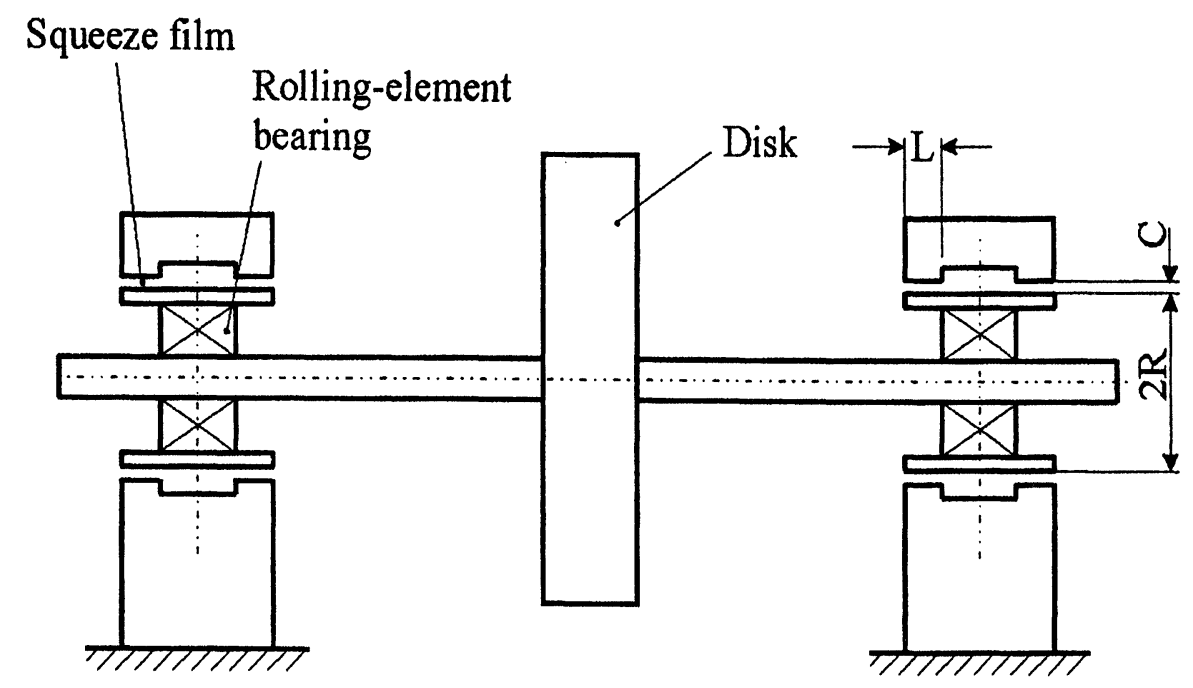

FIGURE 1 Schematic model of a flexible rotor supported on squeeze film dampers without centralized springs. 
Since the rotor system is symmetric about its midplan and the two squeeze film dampers without centralized springs are identical, it is sufficient to consider only one half of the system. Thus, the equations of motion of the rotor system can be expressed in Cartesian coordinates in nondimensional form as follows:

$$
\begin{aligned}
& X_{\mathrm{B}}^{\prime \prime}+\frac{1}{2 \alpha \Omega^{2}}\left(X_{\mathrm{B}}-X_{\mathrm{D}}\right)=\frac{2 B}{\Omega} F_{X}+\frac{W}{\Omega^{2}}, \\
& Y_{\mathrm{B}}^{\prime \prime}+\frac{1}{2 \alpha \Omega^{2}}\left(Y_{\mathrm{B}}-Y_{\mathrm{D}}\right)=\frac{2 B}{\Omega} F_{Y}, \\
& X_{\mathrm{D}}^{\prime \prime}+\frac{1}{\Omega^{2}}\left(X_{\mathrm{D}}-X_{\mathrm{B}}\right)+\frac{2 \xi}{\Omega} X_{\mathrm{D}}^{\prime}=U \Omega^{2} \cos \tau+\frac{W}{\Omega^{2}},
\end{aligned}
$$

$$
Y_{\mathrm{D}}^{\prime \prime}+\frac{1}{\Omega^{2}}\left(Y_{\mathrm{D}}-Y_{\mathrm{B}}\right)+\frac{2 \xi}{\Omega} Y_{\mathrm{D}}^{\prime}=U \Omega^{2} \sin \tau .
$$

Here $X_{\mathrm{B}}, Y_{\mathrm{B}}$ and $X_{\mathrm{D}}, Y_{\mathrm{D}}$ are the displacements of journal and disk stations in the $x$ and $y$ directions, respectively. $\alpha$ is the mass ratio of the rotor system, $\Omega$ the rotor speed ratio, $\xi$ the air damping ratio, $W$ the weight parameter, $U$ the unbalance parameter of the rotor system. $\tau=\omega t$, and $\omega$ is the rotor speed. $\left({ }^{\prime}\right)$ denotes the derivative with respect to the nondimensional time $\tau$ and $B$ is the bearing parameter that includes the effect of oil viscosity and the damper design parameters. $F_{X}$ and $F_{Y}$ are oil-film force components of the squeeze film damper in the $x$ and $y$ directions, respectively. Under the assumption of the short bearing and the cavitated oil film, $F_{X}$ and $F_{Y}$ are (Holmes, 1972)

$$
\begin{aligned}
F_{X}= & -\frac{1}{\sqrt{\left(X_{\mathrm{B}}^{2}+Y_{\mathrm{B}}^{2}\right)}}\left[X_{\mathrm{B}}\left(\varepsilon_{\mathrm{B}} \varphi_{\mathrm{B}}^{\prime} I^{11}+\varepsilon_{\mathrm{B}}^{\prime} I^{02}\right)\right. \\
& \left.-Y_{\mathrm{B}}\left(\varepsilon_{\mathrm{B}} \varphi_{\mathrm{B}}^{\prime} I^{20}+\varepsilon_{\mathrm{B}}^{\prime} I^{11}\right)\right], \\
F_{Y}= & -\frac{1}{\sqrt{\left(X_{\mathrm{B}}^{2}+Y_{\mathrm{B}}^{2}\right)}}\left[Y_{\mathrm{B}}\left(\varepsilon_{\mathrm{B}} \varphi_{\mathrm{B}}^{\prime} I^{11}+\varepsilon_{\mathrm{B}}^{\prime} I^{02}\right)\right. \\
& \left.-X_{\mathrm{B}}\left(\varepsilon_{\mathrm{B}} \varphi_{\mathrm{B}}^{\prime} I^{20}+\varepsilon_{\mathrm{B}}^{\prime} I^{11}\right)\right],
\end{aligned}
$$

where

$$
\begin{aligned}
\varepsilon_{\mathrm{B}} & =\sqrt{X_{\mathrm{B}}^{2}+Y_{\mathrm{B}}^{2}}, \quad \varphi_{\mathrm{B}}=\operatorname{tg}^{-1}\left(\frac{Y_{\mathrm{B}}}{X_{\mathrm{B}}}\right), \\
I^{m n} & =\int_{\theta_{1}}^{\pi+\theta_{1}} \frac{\sin ^{m} \theta \cos ^{n} \theta}{\left(1+\varepsilon_{\mathrm{B}} \cos \theta\right)^{3}} \mathrm{~d} \theta, \theta_{1}=\operatorname{tg}^{-1}\left(\frac{-\varepsilon_{\mathrm{B}}^{\prime}}{\varepsilon_{\mathrm{B}} \varphi_{\mathrm{B}}^{\prime}}\right) .
\end{aligned}
$$

In order to investigate the bifurcation behavior of the rotor system, the nondimensional equations of motion of the system are numerically integrated using a fourth-order Runge-Kutta method with the constant time step size, $2 \pi / 400$, which is so small that there is no significant truncation error in the steady state responses of the rotor system, even if the time step size further reduces. The rotor trajectories, Poincarè map, bifurcation diagram and power spectra are used to illustrate the bifurcation behavior of the rotor system.

\section{Analysis Methods}

First, some basic concepts are introduced. In nonlinear vibration theory, the motion of the rotor system is said to be harmonic or synchronous if the period of the rotor motion is equal to the period of the exciting force. If the period of the rotor motion is equal to $n$th multiple of the period of the exciting force, the motion of the rotor system is $N$-order subharmonic or sub-synchronous. If the ratio of the period of the rotor motion to the period of the exciting force is $q / p$, where $p / q$ is a rational, $p$ and $q$ are integral and there is not a commensurate term between $p$ and $q$, the motion of the rotor system is $p / q$-order sub-super-harmonic or sub-supersynchronous. In the $p / q$ order sub-super-synchronous motion, the orbit of the rotor system will be closed after $q$ whirling cycles, when the rotor system has rotated $p$ cycles. If $p / q$ is an irrational, the motion of the system is generally called almostperiodic, the trajectory will not be closed. If the ratio is not a constant and the trajectory will not be closed, the motion is called nonperiodic or nonsynchronous in the present paper.

Rotor trajectories The most general method in the analysis of the periodic bifurcation behavior 
of nonlinear rotor systems is the observation of rotor trajectories, which consist of the vertical and the horizontal displacements of the rotor system at the same position. However it is difficult to specify the periodic bifurcation behavior of more complex orbits by this method.

Poincarè maps Poincarè maps allow us to reduce the study of a continuous time system (flow) to the study of an associated discrete time system (map), which is often used to distinguish between the periodic and the nonperiodic motions. In order to reduce the influence of the rotor's transient motion, the Poincarè map is used here. When the sampling period of the Poincarè map equals the period of the exciting force, there is only one fixed point in the Poincarè map for the synchronous orbits (period $T$ ) and a set of $N$ discrete fixed points for the $1 / N$-order subsynchronous orbits (period $N T$ ) or $m / N$-order sub-super-synchronous orbits (period $N T / m$, $m<N)$. If the return points of the Poincarè map appear to form a closed curve in the two-dimensional tours, the motion is almost-periodic (quasi$2 \mathrm{H})$ or nonsynchronous, in which the ratio of the rotating frequency of the rotor system to the frequency of the orbit whirling motion is an irrational number. The motion of the rotor system is not periodic in almost-periodic case, but it has only two frequencies in the Fourier spectrum. Finally, if the return points of the Poincarè map do not consist of either a finite set of fixed points or a closed curve, and form several closed (or unclosed) curves or a geometrically fuzzy structure, it means that there are three or more than three dominant incommensurable frequencies in the motion of the rotor system or that the motion of the rotor system is chaotic.

Bifurcation diagrams If the return points of the Poincarè map for any state variable are chosen as vertical coordinate and the rotor speed as horizontal coordinate, the variations of coordinate $Y(n T)$ of the return points in the Poincarè map for the state variable $Y$ with the rotor speed was then plotted to form the bifurcation diagram. In the calculating process, the steady state variables at the end of an integration were used as initial values of the integration at the next speed in order to form a continuous bifurcation diagram. Since the characteristics of the bifurcation diagrams for different variables are similar to each other, we can use the bifurcation diagram of any state variable to show the periodic bifurcation behavior of the rotor system varying with the rotor speed.

Power spectra The power spectrum of the rotor's displacement or velocity only shows the spectrum components of the rotor vibration signals, but it is very useful to distinguish the periodic or nonperiodic motions and the chaotic motions, since the former will have a few sharp peaks in power spectrum, whereas the latter often have a broad spectrum of Fourier components. However, it is impossible to clearly distinguish the sub-synchronous orbits and the almost-periodic orbits in which the whirling frequency of the almost-periodic orbit is approximately equal to that of a sub-synchronous orbit.

\section{RESULTS AND ANALYSES}

Figures 2 and 3 show the bifurcation diagrams for the journal displacement $X_{\mathrm{B}}$ obtained by numerical integration of the differential equations (1)-(4) for

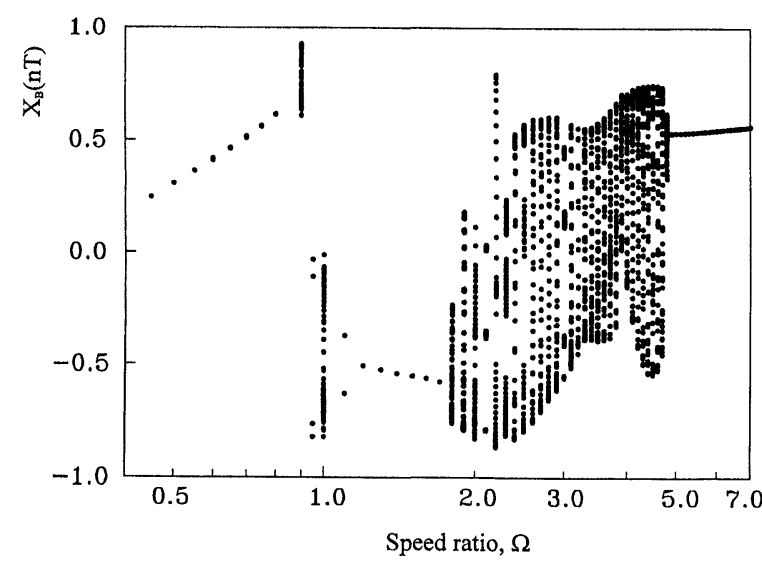

FIGURE 2 Bifurcation diagram of the rotor system, starting with $\Omega=0.0 \quad(\xi=0.0005, \quad \alpha=0.1, \quad B=0.1, \quad W=0.05$, $U=0.6)$. 


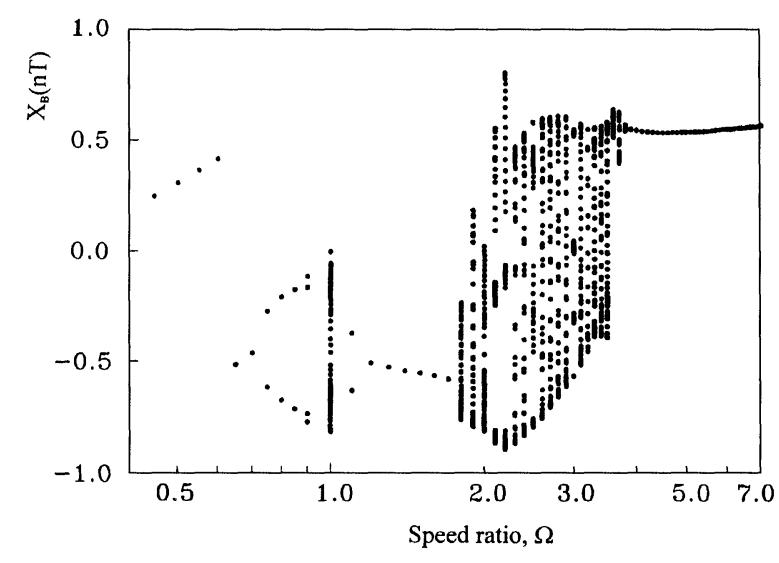

FIGURE 3 Bifurcation diagram of the rotor system, starting with $\Omega=7.0 \quad(\xi=0.0005, \quad \alpha=0.1, \quad B=0.1, \quad W=0.05$, $U=0.6)$.

certain calculating cycles. At each rotor speed, the integration was carried out until the transient response has decayed, i.e., the system had reached its steady state. Generally this took about 50 calculating cycles. The integrating results from 100 to 150 cycles in calculation are outputted as the steady state response of the rotor system at this speed.

Figure 2 was obtained by increasing the rotor speed from $\Omega=0.0$ to $\Omega=7.0$ and using the final state as initial condition for the next rotor speed. The parameters of the rotor system are: $\xi=0.0005$, $\alpha=0.1, B=0.1, W=0.05$ and $U=0.6$. At very low rotor speed $\Omega<0.9$, the motions of the rotor system are synchronous (period $T$ ). At the speed of $\Omega=0.9$, the motion of the rotor system suddenly changes to almost-periodic motion as the return points of the Poincarè map do not coincide to a fixed number of discrete points, but form a closed curve. At $\Omega=0.95$, the motion of the rotor system changes to 1/4-order sub-synchronous (period $4 T$ ), as there are four discrete fixed points in the Poincarè map. At $\Omega=1.0$, the motion of the rotor system again becomes almost-periodic, then bifurcates to 1/2-order sub-synchronous (period 2T) $\Omega=1.1$ from almost-periodic orbit at $\Omega=1.0$. For $1.2 \leq \Omega \leq 1.7$, the motion of the rotor system remains synchronous. For $1.8 \leq \Omega \leq 4.8$, the return points do not coincide to a set of discrete points and the motions of the rotor system in this region are almost-periodic or nonperiodic, the sub-synchronous or sub-super-synchronous orbits only appear at some discrete rotor speeds. For $\Omega$ larger than 4.8 , the motions of the rotor system again are synchronous. In the speed region of $1.8 \leq \Omega \leq 4.8$, the $1 / 3(\Omega=2.05-2.10), \quad 3 / 18(\Omega=2.3), \quad 11 / 40(\Omega=$ $2.605), \quad 3 / 11(\Omega=2.6335-2.635), \quad 1 / 4(\Omega=2.9975)$, $6 / 21(\Omega=3.2), \quad 6 / 23(\Omega=3.51), \quad 1 / 5(\Omega=3.7425)$, $1 / 7(\Omega=4.1485), 6 / 22(\Omega=4.4155)$ order sub-synchronous or sub-super-synchronous orbits are observed and the $7 / 27,1 / 6,1 / 9,4 / 11$ and $1 / 8$-order sub-synchronous or sub-super-synchronous orbits may exist.

Figure 3 shows the results for the same parameters and the same procedure when the rotor speed is decreased from $\Omega=7.0$ down to $\Omega=0.0$. At high rotor speeds, the motions of the rotor system are synchronous and the rotor's orbits are the same as the ones in increasing rotor speed, but the speed region of the synchronous motions in decreasing rotor speed increases to $3.7<\Omega \leq 7.0$ from $4.8<\Omega \leq 7.0$ in increasing rotor speed. At $\Omega=$ 3.7 , the motion of the rotor system suddenly changes from synchronous to almost-periodic, then remains almost-periodic for $1.8 \leq \Omega \leq 3.7$. For $1.2 \leq \Omega \leq 1.7$, the motions of the rotor system are synchronous, so Figs. 2 and 3 show the same results in this region. After $\Omega$ is less than 1.2, the motion of the rotor system bifurcates into different periods. The motion of the rotor system is $1 / 2$-order subsynchronous at $\Omega=1.1$, almost-periodic at $\Omega=1.0$, $1 / 4$-order sub-synchronous at $\Omega=0.9$, and $1 / 2$ order sub-synchronous for $0.75 \leq \Omega \leq 0.85$. When $0.0<\Omega \leq 0.7$, the motions of the rotor system are again synchronous and the rotor's orbits are as same as the ones obtained in increasing rotor speed.

By comparing Figs. 2 and 3, it can be seen that there exist two different operational forms at certain rotor speed regions, i.e., $0.6<\Omega<0.9$ and $3.7<\Omega<4.8$, respectively, which were often called the bistable operations in the rotor systems supported on squeeze film dampers with centralized springs. In the rotor systems supported on squeeze film dampers without centralized springs, the 
motions of the rotor system in the bistable operation speed regions are not always synchronous, but the sub-synchronous, sub-super-synchronous, almost-periodic or nonperiodic motions may coexist at certain speed regions. Whether the rotor system runs into the larger or the smaller orbit within the bistable operation speed regions depends on whether the speed of the rotor system is increased or decreased. Figure 4 shows that the motions of the rotor system for increasing rotor speeds are synchronous, almost-periodic and approximate $1 / 7$-order sub-synchronous at $\Omega=0.8$, 0.9 and 4.1 , respectively, but $1 / 2$-order sub-synchronous, 1/4-order sub-synchronous and synchronous at the same speeds for decreasing rotor speeds. Here the unit circle represents the damper clearance circle, and the sign of the rotor speed indicates whether the results were obtained for increasing $(+)$ or decreasing $(-)$ rotor speed. The unstable operation that exists between the two stable orbits cannot be determined with the numerical integration method used here.

By analyzing the almost- and non-periodic orbits of the rotor system and the Poincarè maps in Figs. 2 and 3 , it is found that the change from the $1 / N$ order sub-synchronous to the $1 /(N+1)$-order subsynchronous motion occurs in two different ways. When $N$ is small, the motion of the rotor system suddenly changes from $1 / N$-order to $1 /(N+1)$ order sub-synchronous motion, and there are no other order sub-synchronous or sub-supersynchronous motions. When $N$ is large, there may exist various sub-synchronous or sub-supersynchronous motions during the change process. The orders of the sub-synchronous or sub-supersynchronous motions occurred between $1 / N$-order
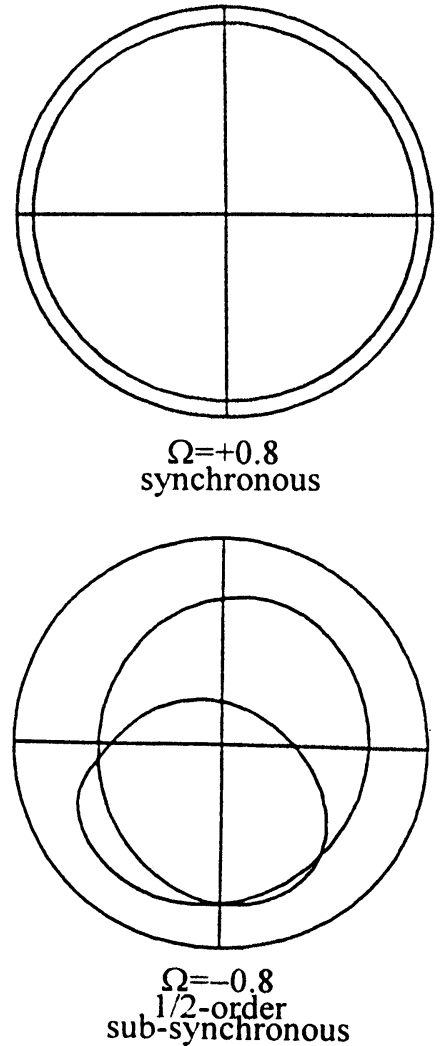
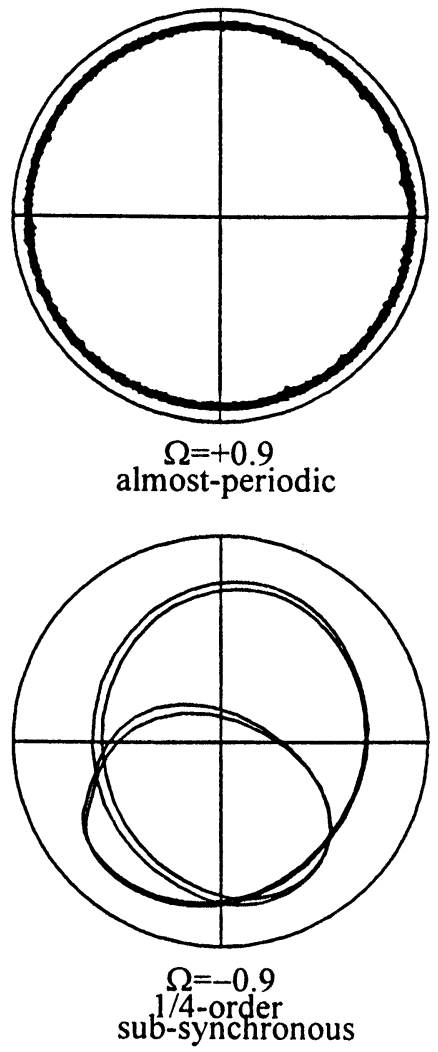

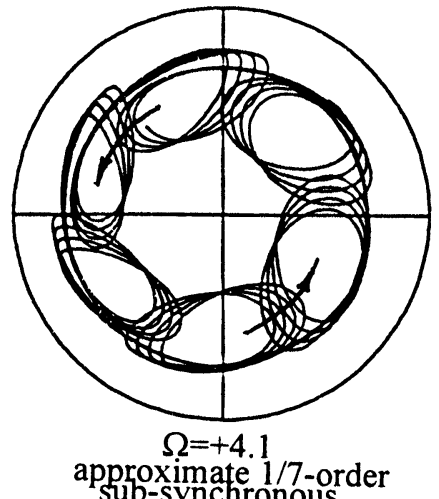

approximate 1/7-orde

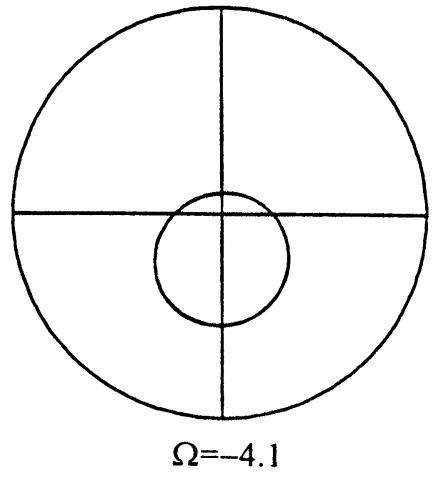

synchronous

FIGURE 4 The typical rotor trajectories in the bistable operations at $\Omega=0.8, \Omega=0.9$ and $\Omega=4.1$. 
and $1 /(N+1)$-order sub-synchronous are basically in agreement with the Farey tree principle observed by Choi and Noah (1994). However, the possibility of the various orders sub-synchronous or sub-supersynchronous motions to occur and the number of the sub-synchronous or sub-super-synchronous motions also depend on the rotor system parameters, especially on the rotor unbalance $U$. The larger the rotor unbalance parameters $U$, the more likely the possibility of the other orders sub-synchronous or sub-super-synchronous motions to occur.

It is very difficult to distinguish the sub-synchronous or sub-super-synchronous motions from the almost- or non-periodic motions, and to determine the rotor speed where the change of the motion order occurs by observing the orbits and the Poincarè maps of the rotor motion. It is found that the sub-synchronous or sub-super-synchronous motions occur between almost-periodic motions whose power spectra are almost identical, and the almost-periodic motions are basically formed by the forward or backward whirling of the smaller order sub-synchronous motion. The sub-synchronous or sub-super-synchronous motion just exists between forward and backward whirling almostperiodic motions. Using this character of the rotor motion, it is easier to distinguish the sub-synchronous or sub-super-synchronous from the complex almost-periodic motions nearby. For example, the motions of the rotor system at $\Omega=+2.9$ and $\Omega=+3.005$ as shown in Fig. 5, can be considered as approximate 1/4-order sub-synchronous motions whose orbits are whirling in backward and forward at a very low frequency, respectively. Their power spectra are very similar. By reducing the considered speed region, the mapping curves in the Poincarè map rapidly converge to almost several distinct points from several arcs. Finally, it is found that there is a $1 / 4$-order sub-synchronous motion at $\Omega=+2.9975$ with return points converging to four discrete fixed points.

From the above analysis, it can be concluded that the almost-periodic motions are more likely to occur than the sub-synchronous or sub-supersynchronous ones. The existence of the almost- periodic motions is the main reason for the fact that the sub-synchronous component of the spectra of the vibration signals in case of nonsynchronous motion is not exactly equal to the integral fraction of the rotor speed frequency observed in experiment and that the orbit of the rotor system is whirling at a very low frequency (Zhu and Feng, 1987).

In developing the Farey tree, there may exist a commensurate term $r$ in fraction $p^{\prime} / q^{\prime}$, i.e., the order of rotor motion is $r p / r q$ order. Although the periods of $r p / r q$ and $p / q$ orders are identical in value, there are different rotor motions associated to them in view of the rotordynamics and their power spectra are also different. In case of the $p / q$ order sub-super-synchronous motion, a closed orbit will be formed after $p$ whirling cycles and $q$ rotating cycles, whereas in case of the $r p / r q$ order sub-super-synchronous motion, a closed orbit will be formed after $r p$ whirling cycles and $r q$ rotating cycles. The rotor orbits, Poincarè maps and power spectra of 3/11- and 6/22-order sub-super-synchronous orbits given in Fig. 6, respectively, very clearly show their differences.

It is also found that the number of fixed points in the Poincarè map for the $m^{\prime} / p$-order sub-supersynchronous motions are the same as that for the $n^{\prime} / p$-order sub-super-synchronous motions, and the number of fixed points in the Poincarè map equals $p$. The sampling period should be $p T$ in order to distinguish $m^{\prime} / p$-order and $n^{\prime} / p$-order sub-supersynchronous motions. If the sampling period is $p T$, there are $m^{\prime}$ and $n^{\prime}$ fixed points in the Poincarè map for the $m^{\prime} / p$-order and $n^{\prime} / p$-order sub-supersynchronous motions, respectively. In fact, the $p$ fixed points of the Poincarè map for $1 / p$-order subsynchronous motions are continuously formed point by point, whereas if the fixed points are spaced by $n$ regular intervals, the motion is $n / p$ order sub-super-synchronous, where $1<n<p$.

Whether the order of the almost-periodic motion is high or low, the return points in the Poincarè map always form a closed curve, but there are different orders for the almost-periodic motion of the rotor system. It is possible to determine the basic order of the almost-periodic motion by analyzing the 
Rotor trajectory
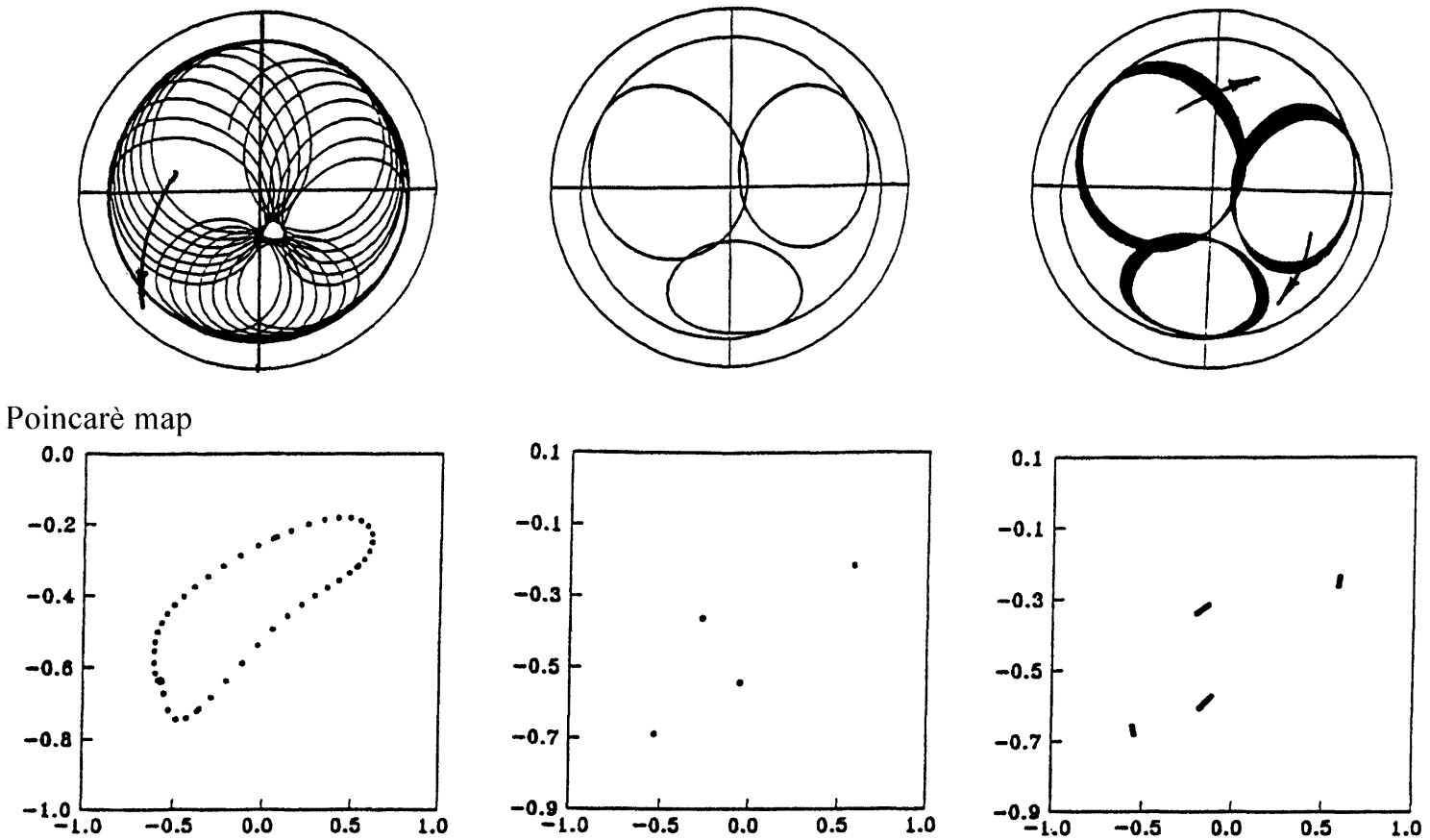

Power spectrum

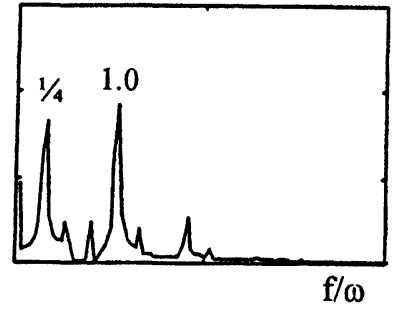

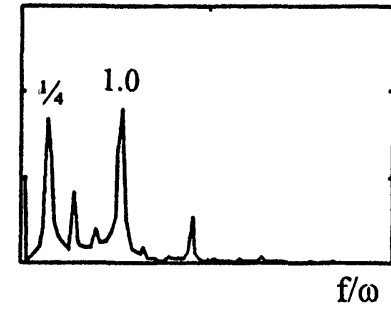

(b)

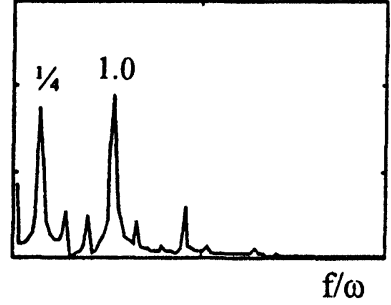

(c)

FIGURE 5 Rotor trajectories, Poincarè maps and power spectra of rotor motions at (a) $\Omega=+2.9$, (b) $\Omega=+2.9975$ and (c) $\Omega=+3.005$.

developing process of closed mapping curve. For example, the closed mapping curve shown in Fig. 7(b) consists of three moving mapping points in Fig. 7(c). Because three points in Poincarè map often stand for 1/3-order sub-synchronous motions, the three moving points basically mean that the rotor system is whirling in sub-synchronous motions with approximate $1 / 3$-order, the orbit of the rotor system is shown in Fig. 7(a). The power spectrum of the rotor motion consists of a 1/3-order sub-harmonic and its multiple components.
The return points in the Poincarè map may form several unclosed curves or several closed curves besides a set of fixed points and a closed curve. Figure 8(a) shows a nonsynchronous orbit with approximate 1/8-order backward whirling, the Poincarè map has eight curves that spirally approach eight fixed points. The motion of the rotor system has two irrationally fundamental frequencies, and their frequency components can be estimated from the power spectrum of the vibration. Figure 8(b) shows a nonsynchronous 
(a)

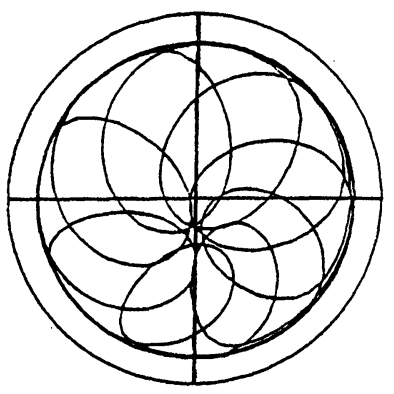

(b)

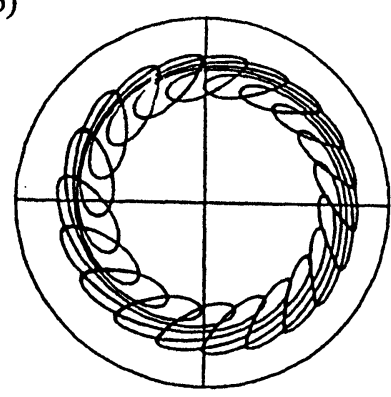

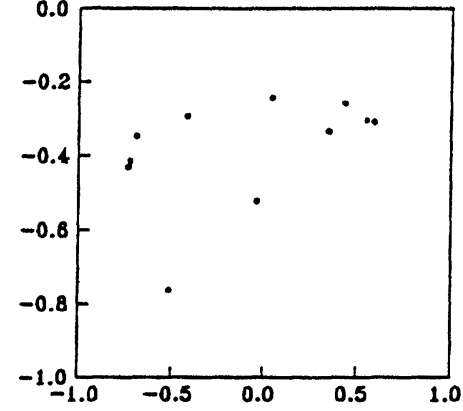

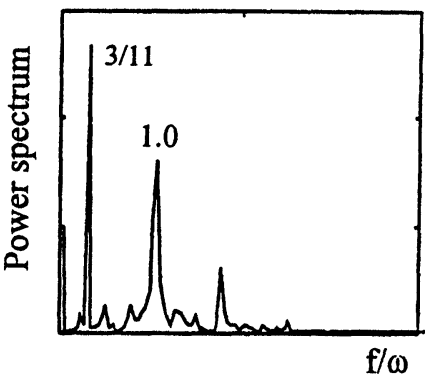

$\mathrm{f} / \omega$
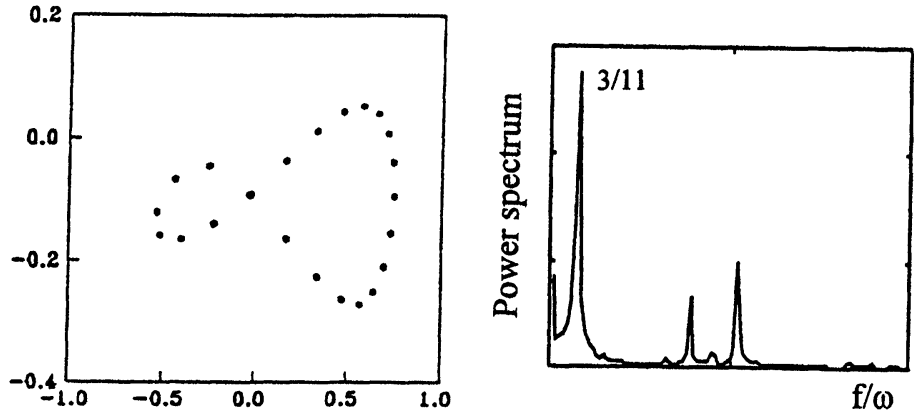

FIGURE 6 Rotor trajectories, Poincarè maps, and power spectra of (a) 3/11- and (b) 6/22-order sub-super-synchronous motions at $\Omega=+2.6335$ and +4.4155 respectively.

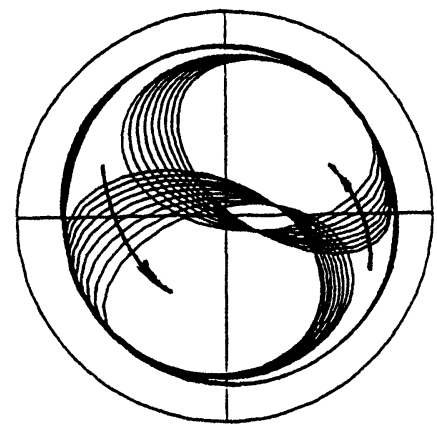

(a) Rotor trajectory

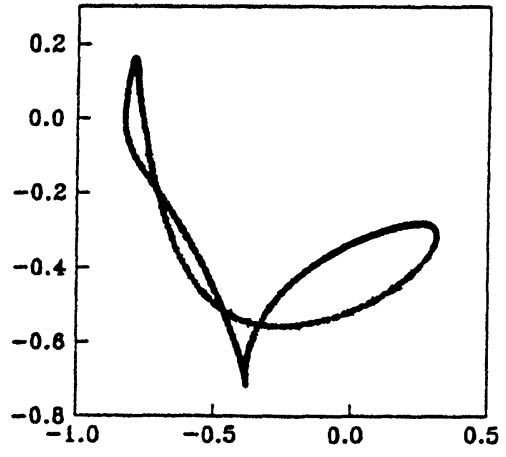

(b) Poincare map for 500 cycles

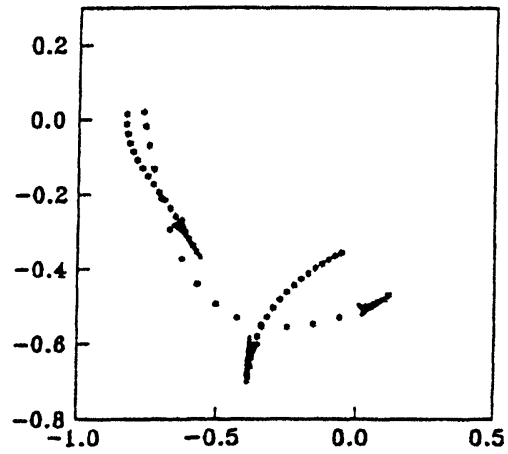

(c) Poincare map for 50 cycles

FIGURE 7 The developing process of almost-periodic motion at $\Omega=+2.9$

orbit with approximate $1 / 2$-order, the Poincarè map has two closed curves. The two closed loops in the Poincarè map often indicate that there are three irrational frequencies (i.e., so-called quit-3H) in the rotor motion. From rotor trajectory, we will find that the rotor system is whirling in forward way at one time, and whirling in backward way at another, and the orbits of the rotor system move 

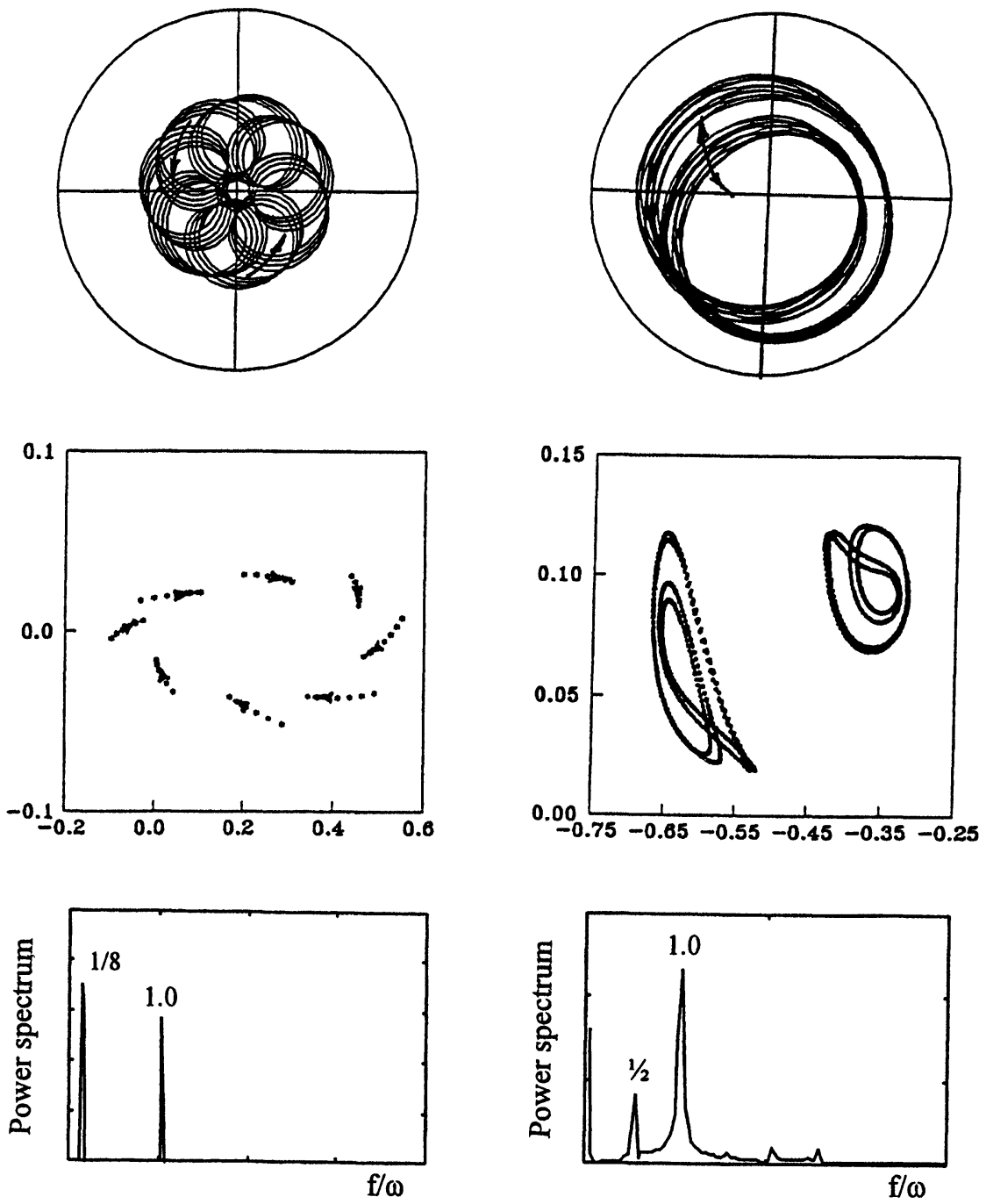

(a)

(b)

FIGURE 8 Rotor trajectories, Poincarè maps, and power spectra of nonsynchronous motions with special characteristics at (a) $\Omega=+4.25$ and (b) $\Omega=-1.05$.

only within certain regions. This is because there are two almost-periodic motions with approximate 1/2-order, which are whirling forward and backward and the amplitudes of these two almostperiodic motions are time-varying. Three peaks in power spectrum indicate that the motion of the rotor system at this speed is approximately $1 / 2$ order sub-synchronous and that the frequency of the change from forward whirl to backward whirl is very slow, which are in agreement with results observed from rotor trajectory.

It can be expected that the chaotic motions found at the rigid rotor supported on eccentrical squeeze film dampers with centralized springs (Zhao and Hahn, 1993) also exist at the flexible rotor on the squeeze film dampers without centralized springs, due to the complexity of the dynamic behaviors of squeeze film dampers without centralized springs. 
The investigation of the chaotic motions will be published in a further paper.

\section{CONCLUSIONS}

1. Analyzing the behavior of the nonlinear rotor system by means of rotor trajectories, Poincarè maps, bifurcation diagrams and power spectra is an effective method to thoroughly understand the complexity of nonlinear rotor systems. It is not enough to investigate the nonlinear behavior only by one of them.

2. There also exist the bistable operations in certain speed regions in the rotor system supported on the squeeze film dampers without centralized springs, which is the same as that in rotor system supported on the squeeze film dampers with centralized springs. In bistable operation speed regions, the rotor system exhibits synchronous, sub-synchronous, sub-super-synchronous and almost-periodic as well as non-periodic motions.

3. The almost-periodic and non-periodic motions more likely appear than the sub-synchronous and sub-super-synchronous motions.

4. The existence of the almost-periodic motions can be used to explain the whirling of whole orbit of rotor system at very low frequency.

5. The bifurcation behavior of the rotor system supported on squeeze film dampers without centralized springs are much more complex than that on the squeeze film dampers with centralized springs and require further investigations.

\section{Acknowledgment}

The first author $(\mathrm{C}, \mathrm{Zhu})$ gratefully acknowledges the support of Alexander von Humboldt Foundation of Germany.

\section{NOMENCLATURE}

$B \quad$ bearing parameter, $B=\mu R L^{3} /\left(m_{\mathrm{B}} C^{3} \omega_{\mathrm{cr}}\right)$

$C$ radial clearance of squeeze film damper unbalance eccentricity

$F_{x}, F_{y}$

nondimensional oil film force

components in $x$ and $y$ directions, respectively

$I^{m n} \quad$ definite bearing integral

$L \quad$ load length of squeeze film damper

$m_{B}, m_{D} \quad$ lumped mass at the journal and mid-plan disk stations, respectively

$R \quad$ radius of squeeze film damper

$t \quad$ time

$U \quad$ unbalance parameter, $U=e / C$

$W \quad$ weight parameter, $W=g / C$

$X, Y \quad$ non-dimensional Cartesian coordinates with $C$

$\begin{array}{ll}\alpha & \text { mass ratio, } a=m_{\mathrm{B}} / m_{\mathrm{D}} \\ \beta & \text { angular position }\end{array}$

$\varepsilon_{\mathbf{B}} \quad$ nondimensional journal eccentricity,

$\varepsilon_{\mathrm{B}}=\sqrt{X_{\mathrm{B}}^{2}+Y_{\mathrm{B}}^{2}}$

$\mu \quad$ oil dynamic viscosity

$\tau \quad$ nondimensional time, $\tau=\omega t$

$\omega \quad$ rotor speed

$\omega_{\mathrm{cr}} \quad$ pin-pin critical speed

$\xi \quad$ air damping ratio

$\Omega \quad$ rotor speed ratio, $\Omega=\omega / \omega_{\mathrm{cr}}$

$\left(^{\prime}\right) \quad$ operator, $\mathrm{d} / \mathrm{d} \tau$

\section{Subscripts}

B bearing

D disk

\section{References}

Adams, M.L. and Abu-Mahfouz, I.A. (1994) Exploratory research on chaos concepts as diagnostic tools for assessing rotating machinery vibration signatures, Proc. IFTOMM, 4th International Conference on Rotordynamics, Sept. 1994, Chicago, pp. 29-39.

Choi, S.K. and Noah, S.T. (1994) Mode-locking and chaos in a Jeffcott rotor with bearing clearances, Journal of Applied Mechanics, 61(1), 131-138.

Holmes, R. (1972) Research note: the nonlinear performance of squeeze-film bearings, J. Mech. Engrg. Science, 14(1), 74-77.

Li, X. and Taylor, D.L. (1987) Nonsynchronous motion of squeeze film damper systems, Journal of Tribology, 109(2), 169-176.

Rabinowitz, M.D. and Hahn, E.J. (1977) Steady state performance of squeeze film damper supported flexible rotors, Journal of Engrg. for Power, 99(4), 552-558. 
White, D.C. (1972) The Dynamics of a rigid rotor supported on squeeze film bearings, I. Mech. E., Conference on Vibrations in Rotating Systems, pp. 213-229.

Zhao, J.Y. and Hahn, E.J. (1993) Subharmonic, quasi-periodic and chaotic motions of a rigid rotor supported by an eccentric squeeze film damper, Proc. Instn. Mech. Engrs., Part C, Journal of Mechanical Engineering Science, 207, 383-392.
Zhu, C.S. and Feng, X.H. (1987) Nonsynchronous orbits observed in rotor-squeeze film damper system, in Rotating Machinery Dynamics, A. Muszynska (Ed.) ASME, New York, pp. 159-165. 


\section{ait \\ ENERGY MATERIALS}

M A N E Y publishing

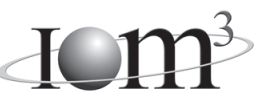

\section{Materials Science \& Engineering for Energy Systems}

Maney Publishing on behalf of the Institute of Materials, Minerals and Mining

The Institute of Materials, Minerals \& Mining

Economic and environmental factors are creating ever greater pressures for the efficient generation, transmission and use of energy. Materials developments are crucial to progress in all these areas: to innovation in design; to extending lifetime and maintenance intervals; and to successful operation in more demanding environments. Drawing together the broad community with interests in these areas, Energy Materials addresses materials needs in future energy generation, transmission, utilisation, conservation and storage. The journal covers thermal generation and gas turbines; renewable power (wind, wave, tidal, hydro, solar and geothermal); fuel cells (low and high temperature); materials issues relevant to biomass and biotechnology; nuclear power generation (fission and fusion); hydrogen generation and storage in the context of the 'hydrogen economy'; and the transmission and storage of the energy produced.

As well as publishing high-quality peer-reviewed research, Energy Materials promotes discussion of issues common to all sectors, through commissioned reviews and commentaries. The journal includes coverage of energy economics and policy, and broader social issues, since the political and legislative context influence research and investment decisions.

\section{CALL FOR PAPERS}

Contributions to the journal should be submitted online at http://ema.edmgr.com

To view the Notes for Contributors please visit: www.maney.co.uk/journals/notes/ema

Upon publication in 2006, this journal will be available via the Ingenta Connect journals service. To view free sample content online visit: www.ingentaconnect.com/content/maney

For further information please contact:

Maney Publishing UK

Tel: +44 (0)113 2497481 Fax: +44 (0)1132486983 Email: subscriptions@maney.co.uk

or

Maney Publishing North America

Tel (toll free): 8662975154 Fax: 6173546875 Email: maney@maneyusa.com

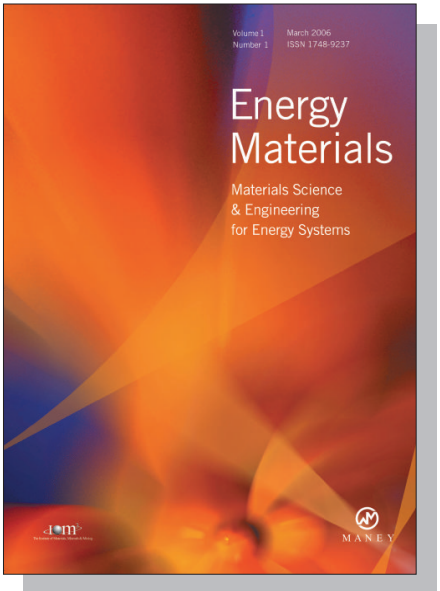

EDITORS

Dr Fujio Abe

NIMS, Japan

Dr John Hald, IPL-MPT, Technical University of Denmark, Denmark

Dr R Viswanathan, EPRI, USA

\section{SUBSCRIPTION INFORMATION}

Volume 1 (2006), 4 issues per year

Print ISSN: 1748-9237 Online ISSN: 1748-9245

Individual rate: $£ 76.00 / U S \$ 141.00$

Institutional rate: $£ 235.00 /$ US $\$ 435.00$

Online-only institutional rate: $£ 199.00 / U S \$ 367.00$

For special $\mathrm{IOM}^{3}$ member rates please email

subscriptions@maney.co.uk

\section{For further information or to subscribe online please visit www.maney.co.uk}



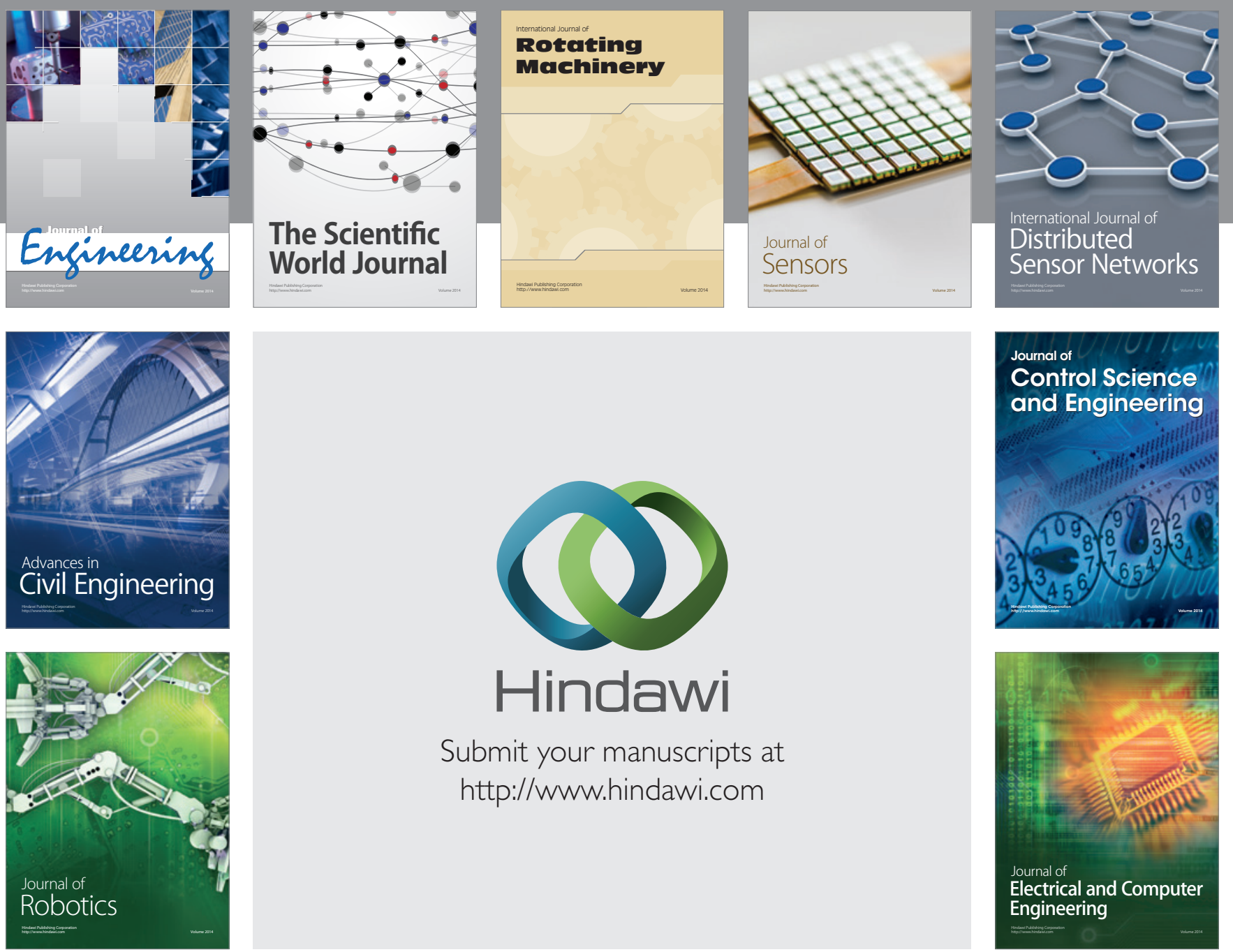

Submit your manuscripts at

http://www.hindawi.com
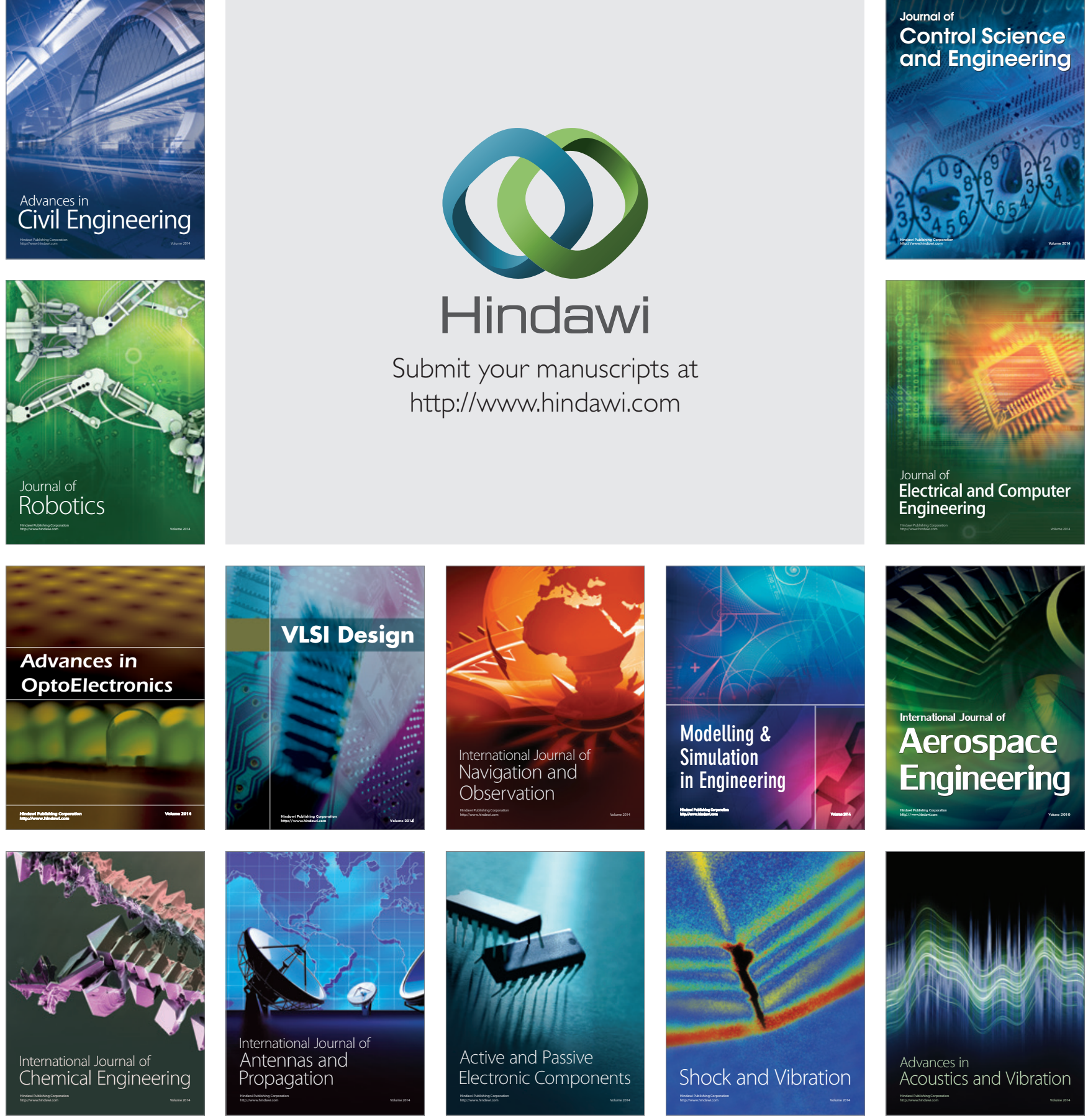